\title{
Lateral Ventricle Ependymal Tumor
}

National Cancer Institute

\section{Source}

National Cancer Institute. Lateral Ventricle Ependymal Tumor. NCI Thesaurus. Code C131594.

An ependymal tumor arising from a lateral ventricle of the brain. 